\title{
A Comparison of the Clinical and Cost- Effectiveness of 3 Intervention Strategies for AIDS Wasting
}

\section{Citation}

Shevitz, Abby H, Ira B Wilson, Ann Y McDermott, Donna Spiegelman, Sarah C Skinner, Kristina Antonsson, Jennifer E Layne, Aaron Beaston-Blaakman, Donald S Shepard, and Sherwood L Gorbach. 2005. "A Comparison of the Clinical and Cost-Effectiveness of 3 Intervention Strategies for AIDS Wasting." JAIDS Journal of Acquired Immune Deficiency Syndromes 38 (4): 399-406. https://doi.org/10.1097/01.qai.0000152647.89008.2b.

\section{Permanent link}

http://nrs.harvard.edu/urn-3:HUL.InstRepos:41384620

\section{Terms of Use}

This article was downloaded from Harvard University's DASH repository, and is made available under the terms and conditions applicable to Other Posted Material, as set forth at http:// nrs.harvard.edu/urn-3:HUL.InstRepos:dash.current.terms-of-use\#LAA

\section{Share Your Story}

The Harvard community has made this article openly available.

Please share how this access benefits you. Submit a story.

\section{Accessibility}




\section{A Comparison of the Clinical and Cost- Effectiveness of 3 Intervention Strategies for AIDS Wasting}

Abby H. Shevitz, MD, MPH, Ira B. Wilson, MD, Ann Y. McDermott, PhD, Donna Spiegelman, PhD, Sarah C. Skinner, MA, Kristina Antonsson, MD, MPH, Jennifer E. Layne, MS, Aaron BeastonBlaakman, MPA, Donald S. Shepard, PhD, and Sherwood L. Gorbach, MD

\section{Abstract}

Objective: To compare oxandrolone (OX) or strength training with nutrition alone (NA) for AIDS wasting.

Subjects: Fifty patients with AIDS; 47 completing the study.

Interventions: Randomization to (1) NA with placebo pills, (2) nutrition with $10 \mathrm{mg}$ of OX administered orally twice a day, or (3) nutrition with progressive resistance training (PRT) for 12 weeks.

Main Outcome Measures: Midthigh cross-sectional muscle area (CSMA), physical functioning (PF), costs, and cost-effectiveness in dollars/quality-adjusted life-years (\$/QALYs).

Results: The OX and PRT subjects had increases in CSMA $(7.0 \% 62.5 \%, \mathrm{P}=0.01 ; 5.0 \% 62.0 \%, \mathrm{P}=$ 0.04 , respectively), although these increases did not differ significantly from the NA arm (NA: $1.0 \%$ 6 1.0\%; OX vs. NA: $\mathrm{P}=0.09$; PRT vs. NA: $\mathrm{P}=0.26$ ). Only PRT caused significant improvements in PF (mean 6 SE: 10.463 .8 points on a 100-point scale) and 7 measures of strength (P values: 0.04 to ,0.001). There were no overall differences between groups in PF change. Among patients with impaired baseline PF, however, OX was significantly less effective than NA and PRT was significantly better than NA. All treatments led to increases in protein intake and performance; NA and PRT also increased caloric intake. The institutional costs per subject in this trial were $\$ 983$ for NA, $\$ 3772$ for OX, and $\$ 3189$ for PRT. At a community-based level of intensity, the institutional costs per QALY were $\$ 45,000$ (range: $\$ 42,000-\$ 64,000$ ) for NA, $\$ 147,000$ (range: $\$ 147,000$ $\$ 163,000$ ) for OX, and $\$ 31,000$ (range: $\$ 21,000-\$ 44,000$ ) for PRT.

Conclusions: OX and PRT induce similar improvements in body composition, but PRT improves quality of life more than nutrition or OX, particularly among patients with impaired PF. PRT was the most cost-effective intervention, and $\mathrm{OX}$ was the least cost-effective intervention.

Key Words: wasting, weight loss, oxandrolone, nutrition, exercise, strength training, muscle, costs, cost-effectiveness 


\section{Introduction}

Wasting has been a prominent feature of HIV infection since its emergence. In the earlier stages of the epidemic, it was associated with extremely high morbidity and mortality, ${ }^{1}$ and it has been an AIDS-defining condition since $1993 .^{2}$ More recently, despite the development of improved therapies for HIV infection and a dramatic reduction in mortality and wasting, ${ }^{3,4}$ weight loss continues to be a problem among infected persons. ${ }^{5}$ It is still strongly related to the risk of disease progression and death, even in the era of highly active antiretroviral therapy (HAART). ${ }^{6,7}$ Of course, wasting remains a devastating problem in most of the world affected by AIDS, where such therapy is not readily available.

Clinical trials have studied strength training and anabolic medications for the treatment of AIDS wasting. In the presence of hypogonadism, testosterone clearly improves weight and lean body mass. ${ }^{9-11}$ Even in HIV-seropositive persons who are eugonadal, testosterone can improve weight and lean body mass ${ }^{12,13}$ and synthetic parenteral androgens also seem to be effective. ${ }^{14,15}$ Most studies of the efficacy of the oral androgen, oxandrolone (OX), have been small, observational, or remain unpublished. ${ }^{16-19}$ The only published randomized clinical trial of OX alone was conducted early in the epidemic for persons with wasting and significant muscle weakness. ${ }^{20}$ In this group with myopathy, weight increased with the use of OX but strength did not and there were no measures of body composition. A dose of $20 \mathrm{mg}$ daily has been used safely for AIDS wasting as reported in 2 abstracts and 1 published report ${ }^{14,17,18}$ and lies at the upper end of the approved doses of this drug for the promotion of weight gain in other medical conditions. The single study comparing $5 \mathrm{mg}$ daily with $15 \mathrm{mg}$ daily for AIDS wasting found weight gain and improved appetite and activity only at the higher dose. ${ }^{20}$ Strength training alone is also effective for restoring weight and lean body mass in HIV wasting, ${ }^{12,13,21}$ but there have been no direct comparisons of exercise with androgens.

Although increased dietary intake is required for weight gain and nutrition intervention is considered the standard of care in the treatment of AIDS wasting, there have been no good studies evaluating their effects on body composition. ${ }^{22,23}$ Clinical trials of pharmacologic and exercise interventions for AIDS wasting have only occasionally included dietary counseling ${ }^{14,20}$ or reported dietary intake. ${ }^{15,21}$ Quality of life has also been neglected in this seriously ill population. To address these gaps in the scientific literature, we conducted a randomized clinical trial comparing OX or progressive resistance training (PRT) with placebo in the setting of intensive nutrition intervention, using midthigh cross-sectional muscle area (CSMA) and self-reported physical functioning (PF) as the major outcomes.

Although other researchers have explored the high cost of other mainstay AIDS interventions, such as HAART, ${ }^{24-27}$ there have been no cost analyses of treatments for wasting, a devastating 
complication, particularly in developing countries with more limited resources. This article includes an analysis of costs of all 3 interventions for the clinical trial as well as for an estimated community-adapted model (ECM) of treatment. The ECM includes 24 rather than 12 weeks of a somewhat less resource-intensive treatment regimen. This study also presents a cost-effectiveness analysis.

\section{METHODS}

\section{Subjects}

Fifty subjects with AIDS wasting were studied in the trial between March 1998 and January 2001 at Tufts University/New England Medical Center in Boston. Subjects were HIV-seropositive and met 1 or more of the following definitions of wasting: (1) documented unintentional weight loss of $10 \%$ from premorbid weight, (2) documented unintentional weight loss of $5 \%$ in the previous 6 months, or (3) body mass index (BMI), $20 \mathrm{~kg} / \mathrm{m}^{2}$. The following were exclusions for participation: (1) excessive vomiting or diarrhea; (2) fever; (3) AIDS-defining complication within 4 weeks before enrollment; (4) corticosteroid, estrogen, progesterone, androgen, anticoagulant, or growth hormone use within the previous 3 months; (5) history of life-threatening reaction to androgens; (6) pregnancy; (7) cardiovascular disease, uncontrolled hypertension, hepatic or renal failure, bleeding disorder, diabetes, breast or prostate cancer, or hypercalcemia; (8) milk allergy; (9) current use of injected drugs; (10) participation in supervised exercise in the previous 4 weeks; (11) physical incapability of exercising; (12) total serum testosterone level ,300 ng/mL in men (unless receiving testosterone replacement); and (13) change in anti-HIV medications within 30 days. All procedures were approved by the Human Investigation Review Committee at New England Medical Center/Tufts University. Informed consent was obtained from participants in accordance with good research practices.

\section{Interventions}

The subjects were randomized to 1 of 3 study interventions for 12 weeks: (1) intensive nutrition intervention with placebo tablets (nutrition alone [NA] arm), (2) intensive nutrition intervention with $20 \mathrm{mg}$ of OX daily (OX arm), or (3) intensive nutrition intervention with PRT (PRT arm). Subjects who were not randomized to the PRT arm spent an additional 30 to 60 minutes weekly with study personnel to provide comparable levels of attention.

\section{Nutrition Intervention}

All participants attended weekly individual visits ranging from 30 to 60 minutes in length. Threeday food records were obtained at baseline, week 6, and week 12 to evaluate dietary intake; 24hour dietary recalls were used if 3-day food records were not feasible. Weekly 24-hour dietary 
recalls were also used to assess intake in the intervening weeks. Dietary recommendations were based on body weight and included higher than maintenance recommendations to achieve weight gain. The goal for total caloric intake was 40 to $50 \mathrm{kcal} / \mathrm{kg} / \mathrm{d}$, and the goal for protein intake was 1.6 $\mathrm{g} / \mathrm{kg} / \mathrm{d}$. Although subjects were encouraged to meet these goals from a variety of food sources, we also supplied and recommended intake of an oral liquid supplement twice daily. Mead Johnson Nutritionals (Evansville, IN) provided Sustacal HN, later remarketed as Boost, each can of which contained $237 \mathrm{~mL}, 240 \mathrm{kcal}, 15 \mathrm{~g}$ of protein, and $3 \mathrm{~g}$ of fat. At weekly sessions, attention was paid to basic nutrition concepts and overcoming individual barriers to good dietary intake, including symptoms, medication schedules, lack of facilities, inadequate social support, and poor access to food. A complete discussion of obstacles encountered and strategies used is published elsewhere. ${ }^{23}$

\section{Drug Intervention}

Study drug was provided by BTG Pharmaceuticals (Iselin, NJ) as 2.5-mg OX tablets and matching placebo tablets; participants took 4 tablets twice daily. At weekly visits, participants in the NA and OX arms were asked about potential side effects, especially priapism and androgenization. Blood was drawn at weeks 6 and 12 to monitor liver function.

\section{Exercise Intervention}

Strength training was performed in a thrice-weekly, one-on-one, periodized program with a research technician, using well-described procedures. ${ }^{28}$ After a warm-up period, 5 exercises were performed: leg press, chest press, knee extension, seated row, leg press, and modified abdominal curl-ups. The first 4 exercises were performed on Keiser pneumatic strength training machines (Keiser Corporation, Fresno, CA), including 3 sets of 8 repetitions each. The workload was progressively increased toward a goal intensity of $80 \%$ of the 1 repetition maximum (1RM) on each machine. 1RMs were retested at sessions 13 and 25 to evaluate progress, and resistance on each machine was adjusted accordingly. Bent-knee abdominal curl-ups were initially performed at 2 sets of 10 repetitions each and were gradually increased in repetitions and, later, with a free weight plate over the chest.

\section{Strength, Power, and Performance Testing}

Muscle strength was assessed by $1 \mathrm{RM}$ testing $^{28}$ on 4 Keiser pneumatic strength training machines: leg press, chest press, leg extension, and seated row. 1RM at baseline was assessed 2 times separated by 1 week, and the best effort was recorded. An isokinetic dynamometer (CYBEX II, Medway, MA) was used to measure strength of the knee extensors and flexors at 60_ per second; data from the nondominant leg were used for analysis. Abdominal muscle endurance was assessed using a modified curl-up (crunch) test, counting the number of curl-ups completed in 1 minute. Physical performance was assessed with a timed 10-repetition chair stand test using standard procedures. $^{29}$ Aerobic endurance was estimated by the 6-minute walk test. ${ }^{30}$ 


\section{Body Composition}

Fasting weight was measured in a hospital gown on a digital scale to the nearest $0.1 \mathrm{lb}$. Whole-body composition was measured with dual-energy x-ray absorptiometry (DEXA) using a Hologic QDR2000 scanner (Waltham, MA) in array mode and was read using accompanying manufacturer software.

\section{Main Outcomes}

Midthigh CSMA was measured from images taken with computed tomography (CT) on a Sytec 4000 CT scanner (General Electric, Milwaukee, WI). The nondominant thigh was imaged in a scout film to identify the full length of the femur. Using fixed bone landmarks at each end of the femur, the length of the femur was measured and bisected. A 10-mm cross-sectional image was taken at the midpoint of the femur using settings of $100 \mathrm{kV}$ and $170 \mathrm{~mA}$ and was recorded digitally. Follow-up films were taken at the identical level of the same leg. The images were analyzed according to optical density on a computer (Macintosh G3; Apple, Cupertino, CA) by a single investigator in a blinded fashion using IMAGE software (version 1.38, National Institutes of Health, Bethesda, MD) modified for quantification of cross-sectional areas of fat, muscle, and bone to the nearest $0.1 \mathrm{~cm}^{2}$.

\section{Physical Functioning}

Subjects were verbally administered the health-related quality-of-life (HRQL) questionnaire used in the HIV Costs and Services Utilization Study (HCSUS) ${ }^{31}$ by trained personnel. A 9-item PF score was coded and analyzed from the relevant items of the questionnaire.

\section{Statistical Methods}

Of the 50 enrolled subjects, 47 participants could be evaluated at 12 weeks and were included in the intent-to-treat analysis. Calculations were conducted with SAS software (version 8; SAS Systems, Cary, NC). Continuous baseline characteristics are described with medians and interquartile ranges (IQRs), and binary characteristics are described with number and percentage. The statistical significance of within-group changes of the 3 treatment arms and of subgroups of interest was assessed by a paired t test.

The statistical significance of differences in the pre-and posttreatment changes within groups were statistically evaluated by linear models with robust variance to allow for valid inference even when the residuals are not normally distributed, ${ }^{32}$ comparing each of the intervention groups (OX or PRT) with the NA group as the referent. Changes in the major outcomes (CSMA and PF) were tested with and without the baseline value for the major outcome and with and without the inclusion of outliers, defined as observations having outcome values falling outside the IQR. The results were similar in all these cases, so the data from models without baseline values but including outlier 
observations are presented. Interactions were tested by comparing models with the interaction term included with corresponding models without the interaction term, using the likelihood ratio (LR) test.

\section{Cost and Cost-Effectiveness Analyses}

Institutional costs for each intervention were calculated by multiplying quantities of each input (personnel or supplies) times their unit costs in year 2000 prices at market value and summing the products. The nutrition intervention cost included 12 counseling sessions, 2 food record collections, and 12 weeks of canned supplements. OX costs included the nutrition intervention costs plus drug. PRT costs included nutrition intervention costs with 3 months of gym fees and 36 personal training sessions. Total costs also included calculation of expenses to the subject and society, namely, transportation and time (opportunity) costs. Investigators developed an ECM, a protocol of these interventions at an intensity level at which community resources or reimbursement would be more feasible, as described by Cisler et al. ${ }^{33}$ Because of their lesser intensity, the interventions were extended from 12 to 24 weeks to achieve theoretically comparable effectiveness to the study protocol. The major differences in the ECM from the study interventions were monthly nutrition counseling and weekly personal training sessions. OX was only proposed for 12 weeks for reasons of safety.

To calculate cost-effectiveness, the HRQL scores were translated into a single health state classification or quality-adjusted life-year (QALY) measure for each client using methods of Brazier et al. ${ }^{34}$

\section{RESULTS}

\section{Baseline Subject Characteristics}

Fifty subjects were enrolled in the study, and 47 completed the 12-week trial, including 33 men and 14 women (Fig. 1). Other screened persons were not enrolled principally for reasons of not meeting weight loss criteria, lack of interest in randomization, recent antiretroviral medication change, or elevated liver function tests. No subjects were rejected because of an inability to tolerate milk, steroids, or exercise. Eighteen subjects were randomly assigned to receive NA, 16 to receive OX with nutrition, and 16 to receive PRTwith nutrition. Of the 2 men and 1 woman who did not complete the study, 2 were in the NA arm and 1 was in the PRT arm.

The study population was ethnically and socioeconomically diverse. Seventy-two percent of subjects reported a household income of less than $\$ 20,000,15 \%$ did not have secure housing, and $52 \%$ were nonwhite. Baseline characteristics of the subjects in the 3 arms are displayed in Table 1. There was a slight imbalance (although not statistically significant, $\mathrm{P}=0.23$ ) of gender among the arms, with women comprising one sixth of subjects in the NA arm and nearly half of the subjects in 
the PRT arm. HAART use, laboratory values, and clinical status were similar among the arms. Fortytwo of the enrollees had a BMI ,20 kg/m². Although BMI was comparable among study arms, initial fat-free mass and muscle area were greatest in the NA arm, which included the greatest proportion of men. Energy intake and self-reported PF were similar among the arms.

\section{Major Outcomes: Cross-Sectional Muscle Area}

As shown in Table 2, CSMA did not change in the NA group, but it increased significantly in the OX group and in the PRT group. The within-group changes represented (mean $6 \mathrm{SE}$ ): 1\% 6 1\%, $7 \% 6$ $2.5 \%$, and $5 \% 62 \%$ of the baseline value, respectively. There was a weak trend toward a difference between the CSMA responses to OX and NA (difference $=5.0 \mathrm{~cm}^{2}, 95 \%$ confidence interval [CI]: 20.6, 10.5; P = 0.09) but not between responses to PRT and NA (difference $=3.3 \mathrm{~cm}^{2}, 95 \% \mathrm{CI}: 21.9$, 8.5; $\mathrm{P}=0.26$ ). Adjustment for gender and HAART did not materially alter these results. We also tested the effect that individuals' baseline values for CSMA may have had on the responses to interventions. The CSMA response to OX seemed to be greatest among those in the lowest tertile of baseline CSMA, whereas the CSMA response to PRT seemed to be similar among all tertiles. The interactions of treatment with baseline CSMA value were not statistically significant (OX vs. NA: $\mathrm{P}=$ 0.19; PRT vs. NA: $\mathrm{P}=0.81$.)

\section{Physical Functioning}

Unadjusted self-reported PF did not change significantly in the NA arm (6.9 65.1 points; $\mathrm{P}=0.20$ ), and the change relative to the subjects' baseline value was $+18.7 \% 616.6 \%$. There was also no significant change in PF among the OX group (21.0 64.7 points or $23 \% 68.7 \%$; $\mathrm{P}=0.83$ ). $\mathrm{PF}$ improved significantly only in the PRT arm (10.4 63.8 points; $\mathrm{P}=0.02)$, an increase relative to the subjects' baseline PF of $+16 \% 66.3 \%$. There were no overall statistically significant differences in the crude PF responses between OX and NA (difference $=28.0$ points, 95\% CI: $221.8,+5.8 ; \mathrm{P}=0.24$ ) or between PRT and NA (difference $=+3.4$ points, 95\% CI: 29.2, 16.1; P = 0.58). Adjustment for gender and HAART use did not substantially change these findings.

Because more subjects than anticipated started with high PF values, we also considered the effect of baseline PF value on response to treatment. The term for interaction between baseline PF and the PRT intervention was statistically significant (OX vs. NA: $\mathrm{P}=0.55$; PRT vs. NA: $\mathrm{P}=0.003$ ). Including interaction terms in the models revealed a net treatment effect of significantly greater improvement with PRT than NA (P, 0.001) and greater improvement with NA than OX $(\mathrm{P}=0.005)$. The modeled responses within each tertile of baseline PF are shown in Figure 2. Changes were small among persons with initially high PF and were greatest among those in the lowest tertile of initial PF. Within each tertile, however, PRT seemed to induce the greatest increases in PF and OX seemed to be least beneficial. In post hoc testing, there was a trend toward greater improvement in PF with PRT than with OX (P = 0.08) and a statistically significant greater improvement in PF with $\mathrm{PRT}$ than with $\mathrm{OX}$ after adjustment for the baseline $\mathrm{PF}$ value $(\mathrm{P}=0.01)$. 


\section{Other Nutrition Outcomes}

Table 2 also presents unadjusted within-group changes in minor outcomes. Total energy intake increased by $36 \%$ of baseline intake among all subjects; the increases in energy intake were statistically significant among subjects in the NA and PRT groups but not in the OX group. Increases in energy intake exceeded the $480 \mathrm{kcal}$ provided by oral supplements by a modest amount in the NA group and by a large amount in the PRT group but not in the OX group. Protein intake increased significantly in all 3 groups, and these increases exceeded the

$30 \mathrm{~g} / \mathrm{d}$ provided by oral supplements. Models that included interactions for baseline values demonstrated a significantly greater improvement with NA than with OX and with PRT than with OX in energy and protein intake. There was no evidence of any increase in gastrointestinal symptoms that could have interfered with intake in the OX group. Together, the 47 subjects achieved a modest but significant average increase in BMI $\left(0.39 \mathrm{~kg} / \mathrm{m}^{2} ; \mathrm{P}=0.01\right)$ and fat-free mass $(1.27 \mathrm{~kg} ; \mathrm{P}, 0.001)$. There were no significant changes in BMI within any treatment arm, however. In the NA and OX arms, fat-free mass increased modestly but significantly $(\mathrm{P}=0.04)$, and there was a trend toward an increase $(\mathrm{P}=0.06)$ in the PRT arm (NA: $0.90 \mathrm{~kg} 61.62$; OX: $1.72 \mathrm{~kg} 6$ 3.00; PRT: $1.17 \mathrm{~kg} 62.24)$.

\section{Strength, Power, and Performance Testing}

Strength and power were evaluated with 4 different strength machine exercises, modified abdominal curl-ups, and two CYBEX II measures. There was a small but statistically significant change only on the curl-up test in the NA arm, and there were modest increases in 1 of the 1RMs and the curl-ups in the OX arm. Improvements on all tests were far exceeded by those in the PRT arm, however, including statistically significant increases in all 4 1RMs, both CYBEX II measures, and the modified curl-ups. There were significant improvements in all groups in the 6-minute walk and repeated chair stand test.

\section{Adherence to the Protocol}

Subjects in all arms demonstrated high levels of adherence to the nutrition and pharmaceutic protocols. They reported drinking almost all of the prescribed liquid supplements (NA: 94\%, OX: 89\%, PRT: $91 \%$ ) and taking the study drug on 92\% of the days in the trial (NA: 94\%, 0X: 88\%). Participants in the nonexercise arms attended 93\% (NA) and 86\% (OX) of their weekly nutrition and monitoring sessions in person. PRT subjects attended an average of $72 \%$ of the 36 possible training visits. 


\section{Adverse Events}

Gastrointestinal symptoms were common during the course of the trial, but there was no evidence of worsening by any intervention. Three people developed opportunistic infections during the study, and 2 more were diagnosed with such conditions shortly after completion of the trial. There were no deaths or serious or unexpected adverse reactions to the study interventions. Serious liver function elevation developed in 2 study subjects and resulted in unblinding of study drug. One had been assigned to OX and had hepatitis $\mathrm{C}$ with unrecognized ongoing alcohol intake; after discontinuation of OX, her laboratory values quickly reverted to baseline. The other subject had hepatitis attributable to efavirenz and had been taking placebo (NA) for the study. Another woman receiving $\mathrm{OX}$ was diagnosed with osteonecrosis of the hip that radiographically antedated her enrollment in the study. There were no reports of hirsuitism, deepening voice, sexual dysfunction, menstrual change (among women), or gynecomastia (among men).

\section{Cost and Cost-Effectiveness}

The calculated institutional cost of OX with nutrition (\$3772) was greater than the cost of PRT with nutrition (\$1636) or NA (\$766) (Table 3). Expectedly, the total costs were higher than institutional costs alone, and the ECM costs were lower than the study costs; however, the interventions retained the same order of costliness. Each of the ECM totals included the following costs for nutrition intervention: $\$ 163$ for 12 weeks of canned nutrition supplements, $\$ 290$ for nutrition counseling, \$34 for food records, and \$109 for the client's time and travel. Although no individual arm demonstrated a large or significant change in QALYs, the increase was greatest in the PRT arm, resulting in the most favorable cost-effectiveness (lowest cost/QALY). Despite including a range of possible costs, the cost-effectiveness estimates did not overlap. Final calculations were substantially least cost-effective for OX.

\section{DISCUSSION}

This is the first study to directly compare nutrition therapy alone with 2 investigational strategies for wasting and the first to test $\mathrm{OX}$ for these outcomes. The results demonstrate that 2 different interventions supported with nutrition, OX and PRT, had comparable effects on body composition in AIDS wasting. In contrast to OX, however, training had a stronger effect on protein and caloric intake as well as strength and a stronger effect on PF, after consideration of baseline functioning, than either of the other 2 interventions. One explanation for the additional PF benefits of the exercise intervention may be that although both groups gained muscle, the quality of the muscle produced may differ between the treatments. This possibility is supported by the absence of strength increase accompanying the muscle mass increase seen with OX. Dietary intervention alone successfully achieved increases in calorie and protein intake (more so than OX) and lean body mass, although the benefits did not translate into measurably increased muscle mass or quality of life. The 
finding that intensive nutrition therapy alone is effective in increasing dietary intake and lean mass is important, given the safety and low cost of this approach.

Although hypogonadal men with HIV infection should clearly be treated with testosterone, ${ }^{9}$ the recommended course of treatment of women and eugonadal men with wasting is still uncertain. Although additional testosterone seems to be of benefit, many patients and providers would prefer an oral androgen if it were safe and effective. The lack of a significant difference between the changes in muscle with $\mathrm{OX}$ and nutrition and the stronger benefits of nutrition and exercise on PF do not support the overall benefit of the drug, however. Although the subjects in our study did not experience any side effects, given our results and the potential toxicity of OX, it is difficult to justify the wide use of this agent for HIV wasting.

We cannot exclude the possibility that some of the prior weight loss experienced by our subjects represented lipoatrophy rather than wasting. Most of our participants had low BMIs, however, and had struggled with weight loss and nausea, anorexia, or diarrhea for years, often since long before beginning HAART. We attempted to verify that weight loss included substantial loss of fat-free mass, which was feasible in subjects who had undergone multiple DXA scans for the Nutrition for Healthy Living (NFHL) cohort study at Tufts University. Data analysis shows that the average fatfree mass of this study's participants was substantially lower than the average fat-free mass of 284 unselected NFHL subjects with DXA scans in 1998 through 2001 (men: 52.7 kg 66.5 vs. 55.8 kg 6 7.0; women: $37.6 \mathrm{~kg} 64.2 \mathrm{vs.} 40.8 \mathrm{~kg} 65.5$ ). This suggests that we successfully selected participants with true wasting and loss of lean body mass, even if they had also experienced fat atrophy.

Our findings differ from others in that earlier studies demonstrated greater success in increasing weight than we did here with OX or exercise. There are several possible explanations for this difference. First, exogenous androgen suppresses endogenous testosterone, theoretically limiting its anabolic effect. Strawford et avoided this potential problem by administering both testosterone and OX, although this is not how OX is used clinically. Increases in muscle mass in this study suggest that the anabolic effect of OX overcame testosterone suppression. Moreover, some of the men in this study were receiving replacement testosterone and could not have had gonadal suppression. It is surprising that $\mathrm{OX}$ with nutrition had less effect on dietary intake and quality of life than did nutrition intervention alone. Possible reasons for this are not known. Although OX still induced a net anabolic effect, perhaps the suppression of endogenous testosterone resulted in decreased wellbeing through mechanisms that are not understood. Lower than anticipated attendance of exercise sessions could also explain some lack of improvement in weight and lean body mass; however, the strength effects of our training were considerable. Overall, there was still a statistically significant increase in weight among all subjects during the course of the study.

Only 1 other study included separate arms with androgens and exercise alone. ${ }^{12}$ In that report, weight, lean body mass, and muscle area seemed to increase more with testosterone than with exercise, although direct comparisons were not reported. The subjects in that trial did not incur increases in strength, however, suggesting that their training was ineffective. Numerous other studies have demonstrated the benefits of exercise; however, effective exercise programs tailored 
for the needs of the wasted HIV-seropositive population are not provided as standard medical care and are typically not covered by medical insurance.

Wasting persists as a major cause of morbidity and mortality in parts of the world without access to antiretroviral agents. ${ }^{8}$ In these places, cost and cost-effectiveness are critical. In the first analysis of its kind, these results show the cost of delivering these interventions to be moderate and the costeffectiveness to be borderline. Although it is not possible from this study to know the separate contributions to the effectiveness of dietary supplements and dietary counseling, the costs of the nutrition intervention were relatively low, including only $27 \%$ for the canned supplements themselves. The most cost-effective treatment was PRT with nutrition intervention, particularly from an institutional perspective, which should encourage the interest of third-party payers. Because the major cost of PRT was the cost of trainers and the opportunity costs of patients, it would be substantially less expensive in low-income countries. OX was the least cost-effective of the interventions, even compared with NA, because it included similar or somewhat greater costs for less of an increase in quality of life. This was true even at a level of intervention reasonably delivered at a community level. Still, compared with other data in the AIDS literature, ${ }^{24-27}$ these costs are approximately 2 to 3 times higher than the cost per QALY gained for providing HAART. Because provision of food is considered standard of care, because this was an effectiveness trial, and because the cost and availability of food are so variable worldwide, the cost of food was not included in our cost analyses. Nonetheless, securing adequate food and/or its cost must be a major focus of providers treating AIDS wasting in resource-poor settings.

This study shows the superiority of exercise over OX in improving PF when there is room for improvement and a dramatic effect on strength, lean body mass, and dietary intake, with a low risk of adverse effects at a lower cost and greater cost-effectiveness. These results should encourage providers, patients, and third-party payers regarding its benefit as a medical therapy. It also confirms the ability of dietary counseling with supplementation to improve nutrition, increasing caloric and protein intake rather than just displacing other sources of energy, and to improve lean body mass.

\section{ACKNOWLEDGMENTS}

The authors extend special thanks to Lynn Bairos, Joan Connors, Jim Fauntleroy, Nkemdiri Iruka, Emily Potts, Stephanie Powers, Ronenn Roubenoff, Nicole Stone, Michael Wood, and the General Clinical Research Center staff, without whom this study could not have been performed. 


\section{REFERENCES}

1. Kotler DP, Tierney AR, Culpepper-Morgan JA, et al. Effect of home total parenteral nutrition on body composition in patients with Acquired Immunodeficiency Syndrome. J Parenter Enteral Nutr. 1990;14:454-458.

2. Centers for Disease Control and Prevention. 1993 revised classification system for HIV infection and expanded surveillance case definition for AIDS among adolescents and adults. MMWR Morb Mortal Wkly Rep. 1992;41(RR-17):1-19.

3. Palella FJ, Jr, Delaney KM, Moorman AC, et al. Declining morbidity and mortality among patients with advanced human immunodeficiency virus infection. HIV Outpatient Study Investigators. N Engl J Med. 1998;338: 853-860.

4. Dworkin M, Williamson J, A.A.S.o.H.D. Project. AIDS wasting syndrome: trends, influence of opportunistic infections, and survival. J Acquir Immune Defic Syndr. 2003;33:267-273.

5. Wanke CA, Silva M, Knox TA, et al. Weight loss and wasting remain common complications in individuals infected with human immunodeficiency virus in the era of highly active antiretroviral therapy. Clin Infect Dis. 2000;31:803-805.

6. Tang AM, Forrester J, Spiegelman D, et al. Weight loss and survival in HIV positive patients in the era of highly active antiretroviral therapy. J Acquir Immune Defic Syndr. 2002;31:230-236.

7. Wheeler DA, Gibert CL, Launer CA, et al. Weight loss as a predictor of survival and disease progression in HIV infection. Terry Beirn Community Programs for Clinical Research on AIDS. J Acquir Immune Defic Syndr Hum Retrovirol. 1998;18:80-85.

8. World Health Organization. WHO report on global surveillance of epidemic-prone infectious diseases-human immunodeficiency virus and acquired immune deficiency syndrome (HIV/AIDS). 2003. Available at: www.who.int/whr/2004/overview/en. Accessed January 6, 2005.

9. Grinspoon S, Corcoran C, Askari H, et al. Effects of androgen administration in men with the AIDS wasting syndrome. A randomized, double-blind, placebo-controlled trial. Ann Intern Med. 1998;129:18-26.

10. Grinspoon S, Corcoran C, Anderson E, et al. Sustained anabolic effects of long-term androgen administration in men with AIDS wasting. Clin Infect Dis. 1999;28:634-636.

11. Miller K, Corcoran C, Armstrong C, et al. Transdermal testosterone administration in women with acquired immunodeficiency syndrome wasting: a pilot study. J Clin Endocrinol Metab. 1998;83:2717-2725.

12. Grinspoon S, Corcoran C, Parlman K, et al. Effects of testosterone and progressive resistance training in eugonadal men with AIDS wasting. A randomized, controlled trial. Ann Intern Med. 2000;133: 348-355.

13. Bhasin B, Storer TW, Javanbakht M, et al. Testosterone replacement and resistance exercise in HIV-infected men with weight loss and low testosterone levels. JAMA. 2000;283:763-770. 
14. Strawford A, Barbieri T, Van Loan M, et al. Resistance exercise and supraphysiologic androgen therapy in eugonadal men with HIV-related weight loss. JAMA. 1999;281:1282-1290.

15. Sattler F, Jaque SV, Schroeder ET, et al. Effects of pharmacologic doses of nandrolone decanoate and progressive resistance training in immunodeficient patients infected with human immunodeficiency virus. J Clin Endocrinol Metab. 1999;84:1268-1276.

16. Earthman C, Reid PM, Harper IT, et al. Body cell mass repletion and improved quality of life in HIV-infected individuals receiving oxandrolone. J Parenter Enteral Nutr. 2002;26:357-365.

17. Fisher A, Abbaticola M, T.O.S. Group. The effects of oxandrolone on the body composition in patients with HIV-associated weight loss. Presented at: Fifth Conference on Retroviruses and Opportunistic Infections, 1998; Chicago, IL.

18. Poles M, Meller JA, Lin A, et al. Oxandrolone as a treatment for HIV-associated weight loss: a one-year follow up. Presented at: Interscience Conference on Antimicrobial Agents and Chemotherapy, 1997; San Diego, CA.

19. Romeyn M, Gunn N. Resistance exercise and oxandrolone for men with HIV-related weight loss [letter]. JAMA. 2000;284:176.

20. Berger J, Pall L, Hall CD, et al. Oxandrolone in AIDS-wasting myopathy. AIDS. 1996;10:16571662.

21. Roubenoff R, McDermott A, Weiss L, et al. Short-term progressive resistance training increases strength and lean body mass in adults infected with human immunodeficiency virus. AIDS. 1999;13: 231-239.

22. Grinspoon S, Mulligan K. Weight loss and wasting in patients infected with human immunodeficiency virus. Clin Infect Dis. 2003;36(Suppl 2): S69-S78.

23. McDermott A, Shevitz AH, Must A, et al. Nutrition treatment for HIV wasting: a prescription for food as medicine. Nutr Clin Pract. 2003;18:86-96.

24. Freedberg KA, Losina E, Weinstein MC, et al. The cost effectiveness of combination antiretroviral therapy for HIV disease. N Engl J Med. 2001; 344:824-831.

25. Holtzer CD, Deeks SG. Impact of HIV-1 protease inhibitors on the cost of treating HIV/AIDS patients. Drug Benefit Trends. 1998;10:27-31.

26. Bozzette SA, Joyce G, McCaffrey DF, et al. Expenditures for the care of HIV infected patients in the era of highly active antiretroviral therapy. N Engl J Med. 2001;344:817-823.

27. Lapins DL, Urdaneta ME, Barrett J, et al. Costs of care for HIV infection in a managed care population from 1995 to 1997. Am J Manag Care. 2000;6:973-981.

28. Baechle T, ed. Essentials of Strength Training and Conditioning. Champagne, IL: Human Kinetics Press; 1994.

29. Studenski S, Perera S, Wallace D, et al. Physical performance measures in the clinical setting. J Am Geriatr Soc. 2003;51:314-322. 
30. American Thoracic Society. ATS statement: guidelines for the six-minute walk test. Am J Respir Crit Care Med. 2002;166:111-117.

31. Hays R, Cunningham WE, Sherbourne CD, et al. Health-related quality of life in patients with human immunodeficiency virus infection in the United States: results from the HIV Cost and Services Utilization Study. Am J Med. 2000;108:714-722.

32. Huber P. The behaviour of maximum likelihood estimators under nonstandard conditions. In: LeCam L, Neyman J, eds. Proceedings of the Fifth Berkeley Symposium on Mathematical Statistics and Probability. Berkeley, CA. University of California Press; 1967:221-233.

33. Cisler R, Holder HD, Longabaugh R, et al. Actual and estimated replication costs for alcohol treatment modalities: case study from Project MATCH. J Stud Alcohol. 1998;59:503-512.

34. Brazier J, Usherwood T, Harper R, et al. Deriving a preference-based single index from the UK SF-36 Health Survey. J Clin Epidemiol. 1998; 15:1115-1128.

35. Fairfield WP, Treat M, Rosenthal DI, et al. Effects of testosterone and exercise on muscle leanness in eugonadal men with AIDS wasting. J Appl Physiol. 2001;90:2166-2171.

36. Roubenoff R, Wilson I. Effect of resistance training on self-reported physical functioning in HIV infection. Med Sci Sports Exerc. 2001;33: 1811-1817. 
Figures

Figure 1: Enrollment in the All-in-ONE trial

79 persons screened in-person

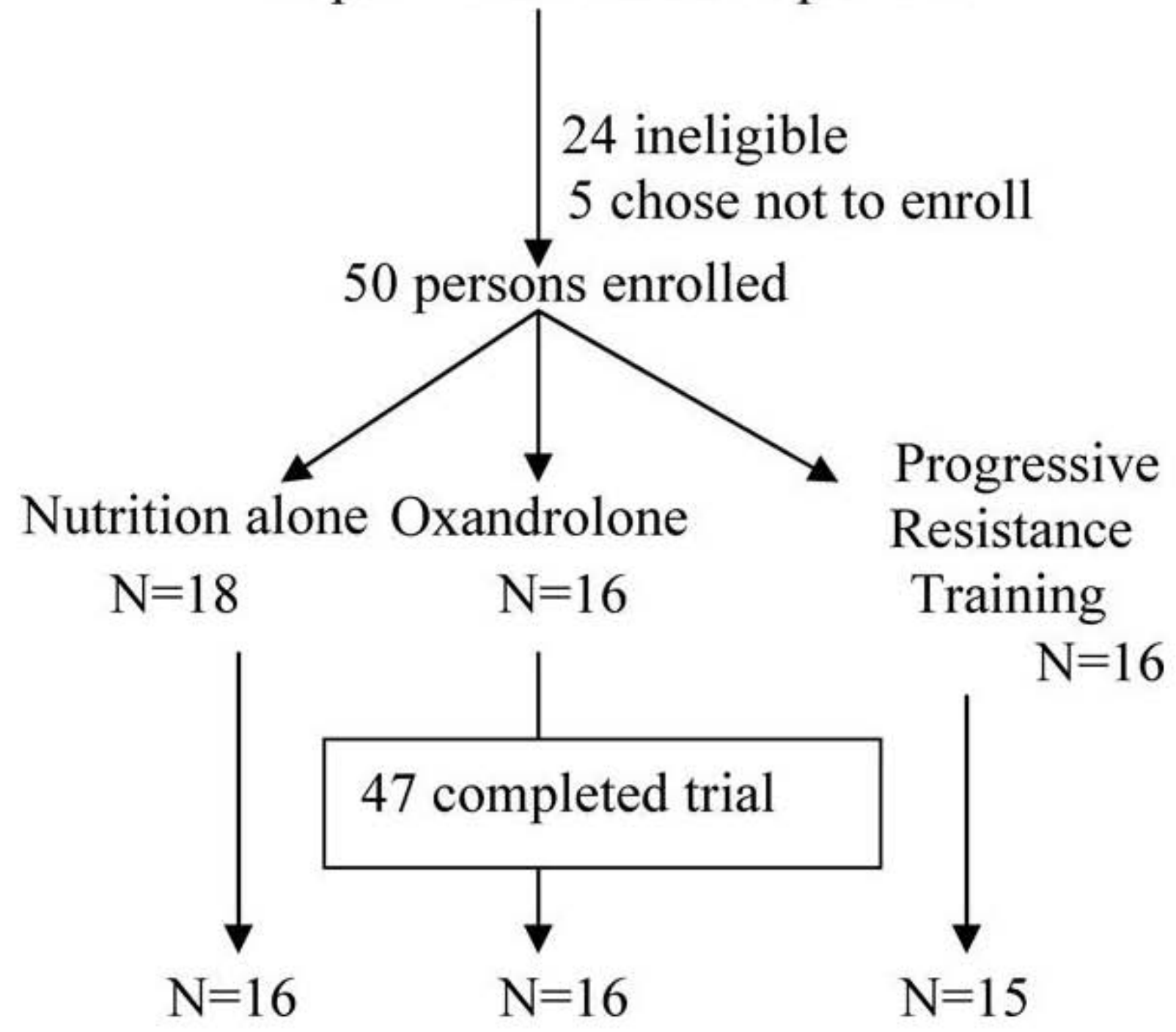


Figure 2: Modeled change in physical functioning (PF) on a 100-point scale by treatment group and baseline PF score.

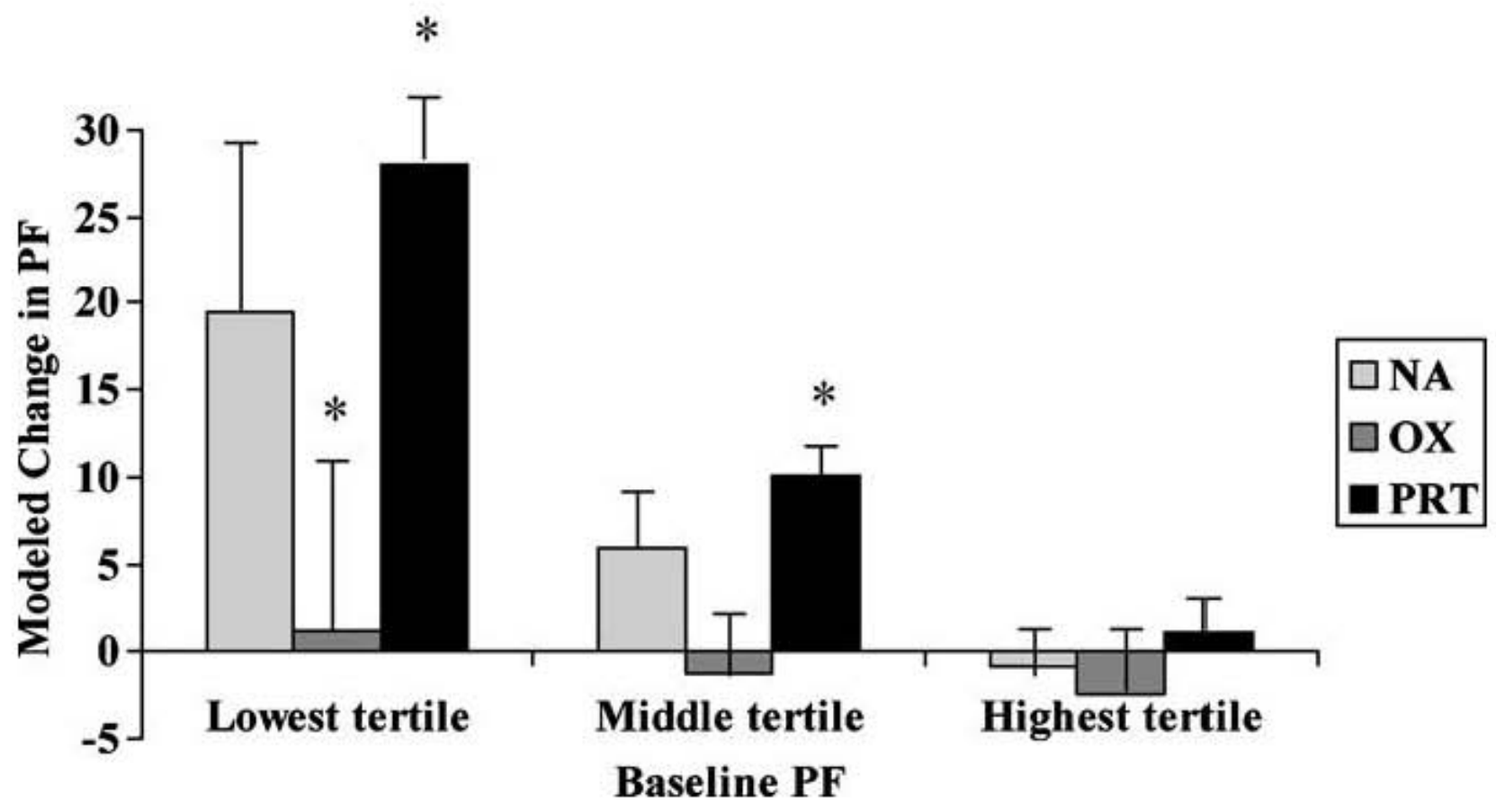

Estimates and SEs are based on models, including the effect of treatment, the effect of the initial PF value, and the effect of their interaction. Baseline PF was grouped as lowest tertile (initial PF between 27 and 73), middle tertile (initial PF between 77 and 89), and highest tertile (initial PF greater than 94 ). ${ }^{*} \mathrm{P}<0.05$ for within-group change. NA indicates nutrition alone arm; OX, oxandrolone arm; PRT, progressive resistance training arm. 


\section{Tables}

Table 1: Baseline Clinical Characteristics, and Nutrition and Physical Functioning Values

\begin{tabular}{lccc}
\hline & NA $(\mathbf{N}=\mathbf{1 8})$ & OX $(\mathbf{N}=\mathbf{1 6})$ & PRT (N = 16) \\
\hline Female gender $(\mathrm{n})$ & $3(17 \%)$ & $5(31 \%)$ & $7(44 \%)$ \\
Age $(\mathrm{y})$ & $43.6(37.3,48.6)$ & $40.8(36.2,45.2)$ & $43.2(38.4,45.9)$ \\
Taking any ART (n) & $15(83 \%)$ & $14(88 \%)$ & $12(75 \%)$ \\
Taking HAART (n) & $13(72 \%)$ & $14(88 \%)$ & $11(69 \%)$ \\
Hepatitis or other AIDS-defining & $6(35 \%)$ & $6(38 \%)$ & $4(27 \%)$ \\
$\quad$ condition $(\mathrm{n})$ & $2(14 \%)$ & & \\
Has hepatitis B $(\mathrm{n})$ & $3(21 \%)$ & $2(14 \%)$ & $2(13 \%)$ \\
Has hepatitis C $(\mathrm{n})$ & $243(198,412)$ & $287(202,601)$ & $3(20 \%)$ \\
CD4 $\left(\right.$ cells $\left./ \mathrm{mm}^{3}\right)$ & $3.2(2.3,3.9)$ & $3.2(2.3,4.0)$ & $3.2(2.3,4.4)$ \\
HIV RNA $\left(\log _{10} / \mathrm{mL}\right)$ & $2705(2296,3594)$ & $2285(1605,3262)$ & $2623(1848,3661)$ \\
Energy intake $(\mathrm{kcal} / \mathrm{d})$ & $108.0(83.0,131.8)$ & $96.9(60.6,115.5)$ & $109.9(75.3,131.6)$ \\
Protein intake $(\mathrm{g} / \mathrm{d})$ & $20.8(19.6,22.8)$ & $19.8(18.0,21.8)$ & $20.6(19.1,21.8)$ \\
BMI $\left(\mathrm{kg} / \mathrm{m}^{2}\right)$ & $53.3(47.2,59.4)$ & $45.3(42.8,53.4)$ & $45.2(35.4,52.1)$ \\
FFM $(\mathrm{kg})$ & $122.3(99.0,152.5)$ & $113.1(94.8,135.4)$ & $110.4(96.8,133.4)$ \\
Muscle area $\left(\mathrm{cm}^{2}\right)$ & $86(72,100)$ & $89(72,100)$ & $89(69,94)$ \\
PF* & $5(28 \%)$ & $5(31 \%)$ & $3(19 \%)$ \\
Initial PF $=100(\mathrm{n})$ & & &
\end{tabular}

$\mathrm{N}(\%)$, median, and IQR are shown.

*Measured on 1-100 scale, with 100 representing best functioning.

ART indicates antiretroviral therapy; FFM, fat-free mass. 
Table 2: Within-Group changes and Between-Group Comparisons of Major and Minor Outcomes; Mean, Standard Deviation, and Probability Value of Change in outcome

\begin{tabular}{|c|c|c|c|c|c|c|c|}
\hline & $\begin{array}{l}\Delta \mathrm{NA} \\
\text { Arm }\end{array}$ & $\begin{array}{l}\Delta O X \\
\text { Arm }\end{array}$ & $\begin{array}{c}\Delta \mathrm{OX} \\
\text { Versus } \Delta \mathrm{NA}\end{array}$ & $\begin{array}{c}\Delta \text { OX Versus } \Delta N A \\
\text { (With Baseline } \\
\text { Interaction) }\end{array}$ & $\begin{array}{c}\Delta \mathrm{PRT} \\
\text { Arm }\end{array}$ & $\begin{array}{c}\Delta \mathrm{PRT} \\
\text { Versus } \\
\Delta \mathrm{NA}\end{array}$ & $\begin{array}{c}\Delta \text { PRT Versus } \\
\Delta N A \text { (With Baseline } \\
\text { Interaction) } \\
\end{array}$ \\
\hline Muscle area $\left(\mathrm{cm}^{2}\right)$ & $2.0(5.3)$ & $6.9(10.0)^{*}$ & $P=0.09$ & $P=0.24$ & $5.3(9.0)^{*}$ & $P=0.26$ & $P=0.63$ \\
\hline $\mathrm{PF} \stackrel{+}{\leftarrow}$ & $6.9(20.5)$ & $-1.0(18.8)$ & $P=0.20$ & $P=0.005$ & $10.4(14.7)^{*}$ & $P=0.59$ & $P<0.001 \S$ \\
\hline BMI $\left(\mathrm{kg} / \mathrm{m}^{2}\right)$ & $0.43(0.90)$ & $0.35(1.25)$ & $P=0.84$ & $P=0.62$ & $0.39(0.94)$ & $P=0.93$ & $P=0.79$ \\
\hline FFM (kg) & $0.90(1.62)^{*}$ & $1.72(3.00)^{*}$ & $P=0.31$ & $P=0.55$ & $1.17(2.24)$ & $P=0.75$ & $P=0.17$ \\
\hline Energy intake (kcal/d) & $542(974)^{*}$ & $403(1078)$ & $P=0.68$ & $P<0.001 \S$ & $724(844) \div$ & $P=0.59$ & $P<0.001$ \\
\hline Energy intake $(\mathrm{kcal} / \mathrm{kg} / \mathrm{d})$ & $8.0(14.3)^{*}$ & $7.0(19.6)$ & $P=0.86$ & $P<0.001 \S$ & $13.3(14.4) \dagger$ & $P=0.35$ & $P=0.04$ \\
\hline Protein intake $(\mathrm{g} / \mathrm{d})$ & $38.6(33.5) \dagger$ & $32.3(36.0) \dagger$ & $P=0.59$ & $P<0.001 \S$ & $40.8(30.7) \dagger$ & $P=0.85$ & $P=0.005$ \\
\hline Protein intake $(\mathrm{g} / \mathrm{kg} / \mathrm{d})$ & $0.60(0.53) \dagger$ & $0.57(0.66) \dagger$ & $P=0.87$ & $P<0.001 \S$ & $0.73(0.54) \dagger$ & $P=0.54$ & $P=0.07$ \\
\hline Leg press (psi) & $34(158)$ & $48(108)$ & $P=0.78$ & $P=0.57$ & $158(132) \dagger$ & $P=0.013$ & $P=0.35$ \\
\hline Chest press (psi) & $-3.3(11.4)$ & $3.8(9.0)$ & $P=0.12$ & $P=0.96$ & $22.0(16.3) \dagger$ & $P<0.001$ & $P=0.005$ \\
\hline Knee extension (psi) & $0.79(8.5)$ & $2.6(7.8)$ & $P=0.62$ & $P=0.10$ & $11.0(11.6) \dagger$ & $P=0.15$ & $P=0.76$ \\
\hline Seated row (psi) & $3.2(13.4)$ & $6.4(9.2)^{*}$ & $P=0.50$ & $P=0.70$ & $16.6(16.5) \dagger$ & $P=0.007$ & $P=0.02 \S$ \\
\hline CYBEX flexion (N-m) & $-0.83(15.3)$ & $3.36(13.41)$ & $P=0.43$ & $P=0.08$ & $9.07(13.7)^{*}$ & $P=0.06$ & $P=0.32$ \\
\hline CYBEX extension $(\mathrm{N}-\mathrm{m})$ & $-1.08(27.2)$ & $-0.29(19.6)$ & $P=0.93$ & $P=0.003$ & $12.6(21.4)^{*}$ & $P=0.11$ & $P=0.03$ \\
\hline Curl-ups (reps) & $3.0(4.7)^{*}$ & $5.6(7.4)^{*}$ & $P=0.35$ & $P=0.51$ & $8.3(9.0) \dagger$ & $P=0.06$ & $P=0.94$ \\
\hline Six-minute walk (yd) & $277(245)^{*}$ & $242(268)^{*}$ & $P=0.74$ & $P=0.03$ & $211(188) \dagger$ & $P=0.51$ & $P=0.004 \S$ \\
\hline Chair stands (s) & $-2.4(2.6) \dagger$ & $-2.9(2.5) \dagger$ & $P=0.64$ & $P=0.14$ & $-3.7(3.0) \dagger$ & $P=0.21$ & $P=0.007 \S$ \\
\hline
\end{tabular}

Statistically significant between-group changes are shown in bold. $* P<0.05 ; \uparrow P<0.01$.

$\div$ Measured on $1-100$ scale, with 100 representing best functioning.

$\S$ Statistically significant $(P<0.05)$ interaction between the baseline value and the response to treatment.

FFM indicates fat-free mass; $\mathrm{N}$-m, Newton-meters; reps, repetitions. 
Table 3: Comparison of Institutional Costs per Subject

\begin{tabular}{lccccc}
\hline & Institutional Study Cost & Total Study Cost & Total ECM Cost & Change in QALYs & ECM Cost/QALY (Range) \\
\hline NA & $\$ 766.34$ & $\$ 983.36$ & $\$ 595.75$ & $0.001(P=0.82)$ & $\$ 45,243(\$ 45,243-\$ 64,335)$ \\
OX versus NA & $\$ 3772.16$ & $\$ 3772.16$ & $\$ 3384.55$ & $0.022(P=0.61)$ & $\$ 146,709(\$ 146,709-\$ 63,163)$ \\
PRT versus NA & $\$ 1636.00$ & $\$ 3189.38$ & $\$ 2987.38$ & $0.045(P=0.20)$ & $\$ 30,708(\$ 21,152-\$ 3551)$ \\
\hline
\end{tabular}

